\title{
Operational, constructive and hydrodynamic parameters of foam devices
}

\author{
Moiseev V. ${ }^{1}$, Manoilo E. ${ }^{2}$, Ponomareva N. ${ }^{3}$, Repko K. ${ }^{4}$, Davydov D. ${ }^{5}$ \\ 1-5. HTPE, NTU “KhPI”, Ukraine, Kharkiv, Kyrpychova str. 2, 61002, , E-mail: bublikova1@gmail.com
}

\begin{abstract}
Industrial implementation of the stabilization method of the gas-liquid layer can significantly expand the field of foam applications and opens new opportunities for intensifying technological processes with the simultaneous creation of low-waste technologies. The basic parameters influencing the hydrodynamics of foam devices are determined, the basic constructions and operating modes of foam devices are considered. The connection of hydrodynamic parameters is revealed. The hydrodynamics of foam layer is considered.
\end{abstract}

Key words: industrial gas emissions; cleaning process; hydrodynamics, mass transfer; foam layer, purification processes; stabilization of foam layer; intensive apparatus

\section{Introduction}

The common method of purifying gas streams is the absorption methods for the absorption of harmful components from the released industrial gases. In this case, either the process of physical absorption occurs, or the absorbent enters a chemical interaction with the absorbable component (the process of chemical sorbtion). One of the promising directions of the intensification of the mass transfer process is the development of apparatus using the principle of interaction of gas-liquid flows in a layer of moving bodies, so-called foam devices with a threephase fluidized bed of an irrigated nozzle

\section{Description of the problem}

The problem of processing industrial gas streams is solved by conducting the process in an intensive mode. The industrial implementation of the absorption processes in the foam layer and the use of the stabilization method of the gas-liquid layer greatly extends the scope of foaming devices and opens up new opportunities for the intensification of technological processes with the simultaneous creation of low-waste technologies. And the use of modern designs of suspended nozzles allows to upgrade existing absorption units.

Usually empty bulbs with a diameter of $20-50 \mathrm{~mm}$ and a density of $100-900 \mathrm{~kg} / \mathrm{m}^{3}$, made of polyethylene, polypropylene, rubber and other relatively light materials, as a rule, were used as nozzle bodies in an absorber with a three-phase fluidized bed. On previously examined nozzles, fluidized bed apparatuses were used mainly in technological cycles with significant specific loads on gas and liquid, which led to high energy consumption. At the same time, in systems for cleaning industrial gases from harmful components present, as a rule, in low concentrations, it is necessary to ensure a high degree of gas purification with minimal liquid flow.

A new volumetric hollow nozzle was developed. The advantage of this nozzle is the transition to a fluidized state at relatively low gas velocities, as well as a developed contact surface of the phases. The funnel structure from which the nozzle is made allows to achieve higher values of the mass transfer coefficients due to the effect of the formation of the film in cells of small size. The nozzle has high porosity and low hydraulic supports. Depending on the material selection, the nozzle can have different wettability.

New nozzles are simple in design and have a relatively low cost, which allows them to be effectively applied in the processes of purifying the gases leaving in different industries, in cooling towers of circulating water supply systems, in a number of other processes of heat and mass transfer. 
Apparatus with a weighted nozzle differs from other by chaotic and pulsating character of movement of the elements of the nozzle in a weighted state. Apparatus with a weighted nozzle can be sectioned, that is, they use different partitions, inserts, stabilizers or grids of a large free section, which divide the section of the apparatus and its working area into separate sections. Such devices successfully solve the problem of large-scale transition from laboratory models to industrial columns without a special change in the efficiency of mass and heat transfer.

The most widespread in the industry are devices with a weighted (fluidized) nozzle, which are constructively simpler and can be improved in the direction of reducing energy consumption, which is important for gas cleaning processes.

Visual observations during the process show the existence of several hydrodynamic modes of operation of devices with a moving nozzle. These regimes are called - initial intermediate, developed (complete) fluidization, stenosis of the apparatus; or - the beginning of fountain (weighing), transition mode, mode of developed turbulence and mode of flooding. In devices of small diameter with a constant value of the mass velocity of a liquid, depending on the speed of gas, four hydrodynamic modes can be distinguished. In the mode of initial pseudosuppression there is a "fountain": they pass into the fluidized state of the nozzle bodies only in the central part of the apparatus, remaining stationary under the walls. The liquid flows down the surface in the form of a film. In the mode of intermediate fluidization, the body bodies located near the walls begin to move down and pass into the central part of the apparatus. In the mode of developed (full) fluidization, all nozzle bodies are in a fluidized state, circulating inside the apparatus: in the central part the movement is directed upwards, and under the walls - downward. The amount of liquid contained in the apparatus increases and it is present mainly in the form of droplets or in the composition of the gas-liquid structure, which is close to the bubble layer.

Together with the subsequent increase in gas velocity, the amount of fluid retained in the layer increases, which, in the end, leads to the flooding of the apparatus.

In addition, on the hydraulic resistance of the device with a three-phase layer, the resistance of the support-distributing plate is significantly affected by the hydraulic resistance of the apparatus: the less the free cross section of the plate, the greater the deviation in the graph of the dependence of the hydraulic resistance on the speed of gas in the apparatus from the horizontal line in the mode of developed fluidization.

\section{Conclusion}

An analysis of a number of studies [1-2] shows that the promising direction of the intensification of the mass transfer process is the development of apparatuses with a three-phase fluidized bed of irrigated nozzle of complex forms with mesh materials. For gas cleaning systems from gaseous components, it is necessary to provide small loads on the liquid while maintaining a high degree of purification. This can be achieved by using a drip tray with a small free section and a newly designed nozzle.

\section{References}

[1] V. F. Moiseev, E. V. Manoylo, K. Yu. Repko, D. V. Davydov. Treatment of gas-discharge systems on tubular lattices with foam layer stabilizer. Bulletin of NTU "KhPI", № 53, pp. 114123, 2017, https://doi.org/10.20998/2413-4295.2017.53.17

[2] V. F. Moiseev, E. V. Manoilo, N. G. Ponomaryova, K. Yu. Repko, D. V. Davydov. Methodology of calculation of construction and hydrodynamic parameters of a foam layer apparatus for mass-transfer processes. Bulletin of NTU "KhPI", № 16, pp. 165 - 176, 2018, https://doi.org/10.20998/2413-4295.2018.16.25 